Chapter 26

\title{
Modeling of Proppant Permeability and Inertial Factor for Fluid Flow Through Packed Columns
}

\author{
Bruce R. Meyer, Lucas W. Bazan and Doug Walls \\ Additional information is available at the end of the chapter
}

http://dx.doi.org/10.5772/56299

\begin{abstract}
Standard industry testing procedures provide proppant quality control and methods to determine long term reference conductivity for proppants under laboratory conditions. However, test methods often lack repeatable results. Additionally, the testing procedures are not designed to account for fundamental parameters (e.g., proppant diameter, porosity, wall effects, multi-phase/non-Darcy effects, proppant and gel damage) that greatly reduce absolute proppant bed conductivity under realistic flowing conditions.
\end{abstract}

A constitutive model for permeability and inertial factor for flow through packed columns has been formulated from fundamental principles. This work provides a detailed deterministic proppant permeability correlation and defines a methodology to help explain why different proppant types behave differently under stress. The theory also characterizes the origin of inertial, or non-Darcy flow, based on a unique approach formulated from the extended Bernoulli equation based on minor losses. The physical model provides insight into the dominant parameters affecting the pressure drop in a proppant pack and improves our understanding of fluid flow and transport phenomena in porous media.

The fundamental solution for flow through packed columns can be characterized by the sum of viscous (Blake-Kozeny) and inertial forces (Burke-Plummer) in Ergun's equation. Coupling Ergun's equation with the Forchheimer equation results in a deterministic set of equations that describe the fracture permeability and inertial factor as functions of the proppant diameter, pack porosity, sphericity, and fracture width. Plotting the dimensionless permeability, $\left(\mathrm{k} / \mathrm{d}_{\mathrm{p}}\right.$ ${ }^{2}$ ), versus the characteristic proppant porosity parameter, $\Omega$, is a very useful diagnostic tool that can indicate: 1) sphericity, 2) channeling, 3) crushing, 4) non-uniform sphere size distribution, 5) embedment and 6) deviation of the friction multiplier $\lambda_{m}$ from Ergun's equation. 
The dimensionless experimental proppant permeability data can be plotted as a linear function of dimensionless porosity with large deviations from these equations signifying poor or inconsistent experimental results or inadequate proppant characterization. The formulated permeability and non-Darcy equations provide the foundation for a quantitative (including quality control of the test) and qualitative analyses for determining fracture permeability and the inertial factor based on the physical properties of the proppant pack.

\section{Introduction}

Hydraulic fracturing has been the major and relatively inexpensive stimulation method used for enhanced oil and gas recovery in the petroleum industry since 1949. The primary goal of a hydraulic fracture treatment is to create a highly conductive flowpath for hydrocarbon production. Fracture conductivity is defined as the product of the packed bed width and permeability. An ideal fracture would possess infinite conductivity. However, producing proppant packs have finite permeability and conductivity. Proppant beds are also subjected to damage and conductivity degradation over time including proppant embedment, formation spalling, temperature degradation, non-Darcy flow, multiphase flow, non-uniform proppant distribution, cyclic stress, gel damage, fines migration, and other effects (Palisch et al., 2007).

The American Petroleum Institute (API) developed conductivity testing procedures outlined in API RP-61 to provide a methodology for consistent and repeatable results. The testing conditions include using the Cooke Conductivity Cell with steel pistons loaded at $2 \mathrm{lb} / \mathrm{ft}^{2}$ at ambient temperature. The stress measurements are maintained for 15 minutes with $2 \% \mathrm{KCl}$ fluid pumped at a rate of $2 \mathrm{ml} / \mathrm{min}$. An industry consortium proposed changes to API RP-61 to replace the steel pistons with Ohio Sandstone, increase the testing temperature to $150^{\circ} \mathrm{F}$ or $250^{\circ} \mathrm{F}$ and maintain the stress for 50 hours. The modified API RP-61 is referred to as "long-term" conductivity, is accepted as the standard testing procedure for proppant, and has been adopted by the International Organization for Standardization (ISO) as ISO 13503-5. The original API RP-61 method is referred to as "short-term" conductivity testing. These testing procedures provide proppant conductivity under laminar (baseline or reference) conditions but fail to predict realistic fracture conductivity under flowing conditions because the tests do not account for the permeability reduction because of proppant pack damage mechanisms. There is tremendous superficial velocity insidea producing hydraulic fracture resulting in significantenergy loss from the kinetic and viscous energy losses and hydrocarbon inertial effects. The constitutive parameters determining the pressure losses are the rate of fluid flow, viscosity and density of the fluid, size, shape, packing orientation and surface of the proppant. In petroleum engineering for a single phase fluid, the energy loss is typically described by a form of the Forchheimer equation (Eq. A. 20) as a sum of the Darcy and non-Darcy pressure drops

$$
-\frac{d p}{d x}=\frac{\mu}{k} v+\beta \rho v^{2}
$$


where the first term on the right hand side of this equation represents the viscous effects and the second term the inertial or minor loss effects. Multiphase fluid interaction (gascondensate, oil-water, etc.) causes pressure losses as multiple viscosities move through the proppant pack at different velocities (fluid mobility). The non-Darcy beta factor, $\beta$, is a material property of proppant that quantifies the inertial or minor losses as a result of fluid contraction and expansion. The greater the inertial losses, the greater the beta coefficient which increases the total pressure loss in the proppant pack. The effects of the beta coefficient can be reduced by increasing the porosity and permeability of the proppant pack, reducing the mesh distribution, and by using more spherical proppant with lower surface friction. Proppant crush tests are one method to determine some of these physical proppant parameters under in-situ conditions.

Standardized crush test procedures are outlined in API RP-56, RP-58 and RP-60 and are summarized in ISO 13503-2. The intent of these tests is to provide a comparison of the physical characteristics of various proppants including crush test results. Again, there are limitations of the testing methodology that do not simulate actual conditions within a producing fracture. However, the actual testing methods, specifically the loading of the cell, can be even more immediately problematic to results. Results from eleven different companies testing a common sample of 16/30 Brown Sand indicate varying test results between companies as high as $25 \%$ (Palisch et al., 2009).

This work provides a detailed deterministic proppant permeability correlation and presents a methodology to help explain why different proppant types behave differently under stress. The governing equations for flow through pack columns are formulated in Appendix A. Derivation of the theoretical fracture permeability and inertial coefficient, $\beta$, are also given in Appendix A.

\section{Pressure loss equations for flow through packed columns}

This section summarizes the equations for viscous and inertial flow in packed columns and presents a correlation model for fracture permeability. The flow through packed columns may be characterized as the sum of frictional (viscous) and inertial (minor losses) forces. The governing pressure loss equation from Eq. A.18

$$
-\frac{d p}{d x}=\frac{72 \lambda_{m}(1-\phi)^{2} \mu v}{\phi^{3} d_{p}^{2}}+\frac{3}{2} f_{0} \frac{1-\phi}{\phi^{3}} \frac{\rho v^{2}}{2 d_{p}}
$$

where from experimental data $\lambda_{m}=25 / 12$ and $f_{0}=7 / 3$. Viscous forces dominate laminar flow regimes while kinetic forces dominate inertial flow. Ergun developed his famous equation for the total pressure loss in packed columns for all flow regimes by simply adding the BlakeKozeny equation for viscous dissipation and the Burke-Plummer equation for inertial losses. Placing Eq. 2 in terms of dimensionless groups we have (see Eq. A.19) 


$$
-\left(\frac{d p}{d x} \frac{d_{p}}{\rho v^{2}}\right)\left(\frac{\phi^{3}}{1-\phi}\right)=\frac{150(1-\phi)}{\operatorname{Re}}+\frac{7}{4}
$$

This is the Ergun equation (see Bird 1960) where $\operatorname{Re}=\rho v d_{p} / \mu, \lambda_{m}=25 / 12$ and $f_{0}=7 / 3$ have been substituted. To account for proppant sphericity, the particle diameter in the above equations can be replaced by $\left(\Phi d_{p}\right)$. Figure 1 shows the general behavior of the Ergun equation on a log-log plot with the Blake-Kozeny and Burke-Plummer equations for reference.

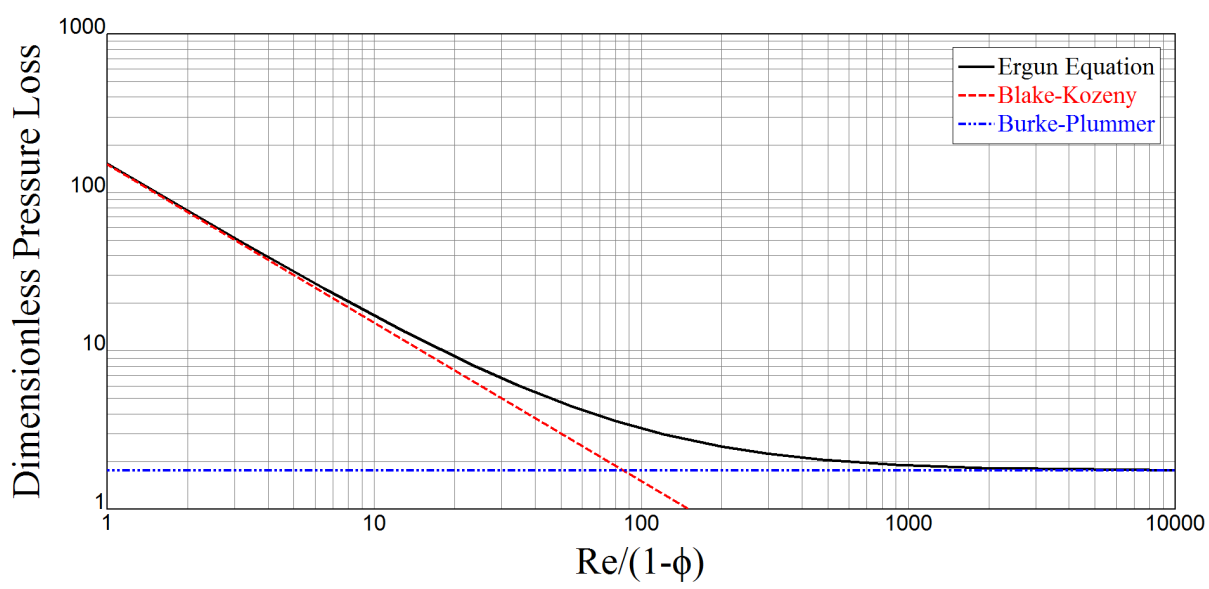

Figure 1. The Ergun equation with the Blake-Kozeny and Burke-Plummer equations.

Rearranging the Forchheimer equation (Eq. 1) into dimensionless groups (see Eq. A.21) we find

$$
(-d p / d x) / \beta \rho v^{2}=1 / \operatorname{Re}_{\beta k}+1
$$

where $\operatorname{Re}_{\beta k}=\rho v \beta k / \mu$. Multiplying Eq. 4 by $7 / 4$, replacing $\operatorname{Re}_{\beta k}$ with $\operatorname{Re}$, and $\beta$ and $k$ in terms of the proppant diameter and porosity (see Eq. A.24) one can show that it is identical to Ergun's equation (Eq. 3). 


\section{Proppant permeability formulation}

The formulation of the proppant permeability (and inertial factor) is presented in Appendix A. It can be shown (see Eq. A.23 through Eq. A.35) that the dimensionless proppant permeability in terms of the proppant diameter, porosity, slot width, and sphericity is

$$
\frac{k}{d_{p}^{2}}=\Omega \Psi
$$

where

$$
\begin{gathered}
\Omega=\frac{\phi^{3}}{72 \lambda_{m}(1-\phi)^{2}}\left(1+a_{D}\right)^{-2} \\
\Psi=\Phi^{2}\left(\frac{1+a_{D}}{1+\Phi a_{D}}\right)^{2} \\
a_{D}=\frac{a_{w}}{a_{s}}=\frac{d_{p}}{3(1-\phi) w}=\frac{d_{p} \rho}{3 C_{a}}
\end{gathered}
$$

Thus if the experimental proppant permeability data is fitted with Eq. 5, the dimensionless permeability $\left(k / d_{p}^{2}\right)$ should be a linear function of the characteristic proppant pack parameter $(\Omega)$ with the slope represented by the proppant sphericity-specific surface area parameter $(\Psi)$. The proppant sphericity can then be found from the slope using Eq. 7

$$
\Phi=\frac{\sqrt{\Psi}}{1+a_{D}(1-\sqrt{\Psi})}
$$

The above equation works well for determining the proppant sphericity provided that the friction multiplier is a constant for all bed packing (i.e., $\lambda_{m}=25 / 12$ ), the proppant sphere size is uniform, and that the sphericity ( $\Phi)$ is a constant. But in reality, $\Phi$ is generally a function of $\Omega$, (i.e., $\Phi=f(\Omega)$ ). Pan et al. (2001) proposed a four parameter model to correlate permeability with porosity and sphere size distribution for random sphere packing. However, plotting dimensionless permeability $k / d_{p}^{2}$ versus $\Omega$ is a very useful diagnostic tool. Large deviations can signify poor or inconsistent experimental results, inaccurate calculation/measurement of the mean proppant diameter (especially for slopes greater than unity), or proppant porosity 
(and width) measurement errors as a function of closure. A diagnostic plot of $k / d_{p}^{2}$ versus $\Omega$ will provide insight into the topics discussed above and also provide a comparison of different proppants and their relative pack permeability as closure stress increases (i.e., low values of $\Omega$ ). The main emphasis of this paper is not to provide a detailed deterministic proppant permeability correlation but rather to provide a methodology to help explain and understand why different proppant types behave differently under stress.

Although Eq. 5 is a very good correlation for diagnostics, other forms of this equation (e.g., $k / d_{p}^{2}=a_{0}+a_{1} \Omega+a_{2} \Omega^{2}$ or $k / d_{p}^{2}=a \Omega^{\alpha}$ ) also fit the data very well over limited ranges for some proppants. The other major advantage of correlating the permeability data with $\Omega$ is that $\Omega$ has the correct limits for mono-layers (i.e., as $\phi \rightarrow 1, \Omega \rightarrow w^{2} /\left(d_{p}^{2} 12\right)$ ). Figures 2 and 3 illustrate images for a 20/40 Northern White Sand and a 20/40 Brown Sand, respectively. The Northern White has a sphericity of about 0.73 while the Brown Sand has a much lower sphericity of about 0.5 . Sintered bauxite and resin coated sands have much higher sphericity of approximately 0.90 and $0.80-0.85$ respectively as illustrated in Figures 4 and 5.

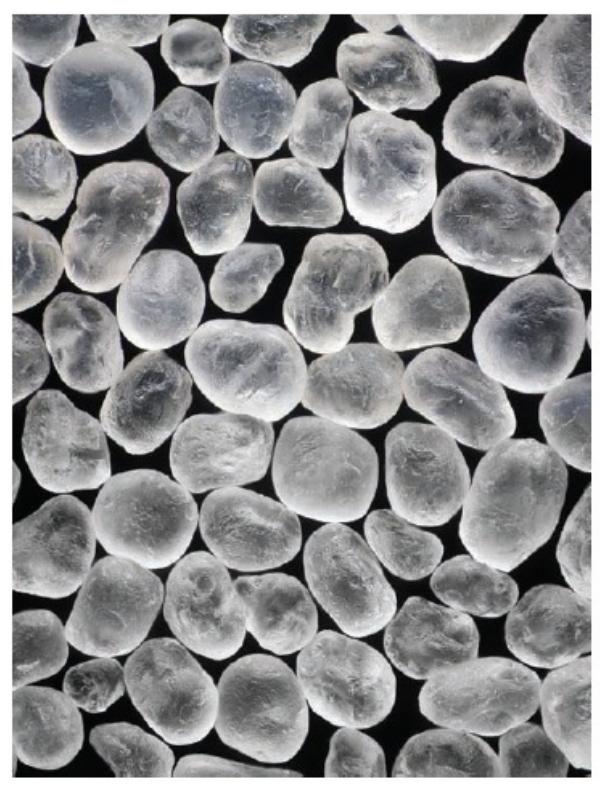

Figure 2. Northern White Sand, sphericity 0.73 - Photo courtesy: Santrol. 


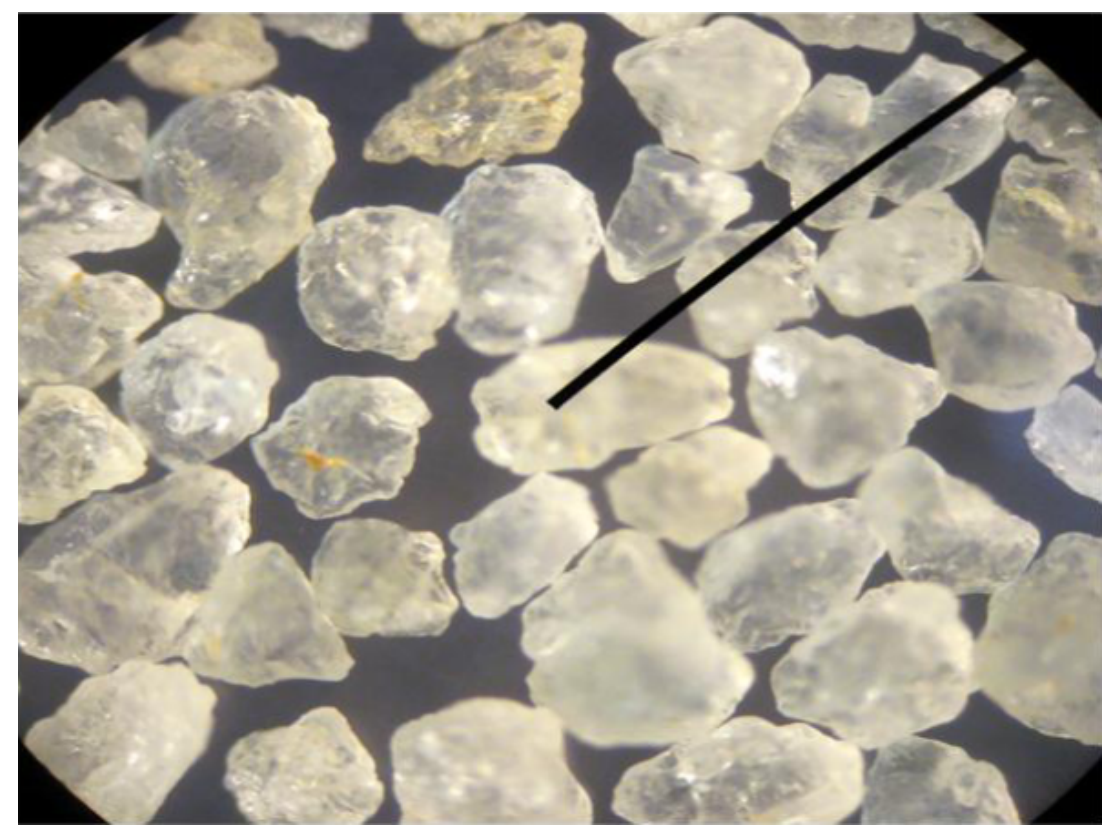

Figure 3. Brown Sand, sphericity 0.50 - Photo courtesy: U.S. Silica Company.

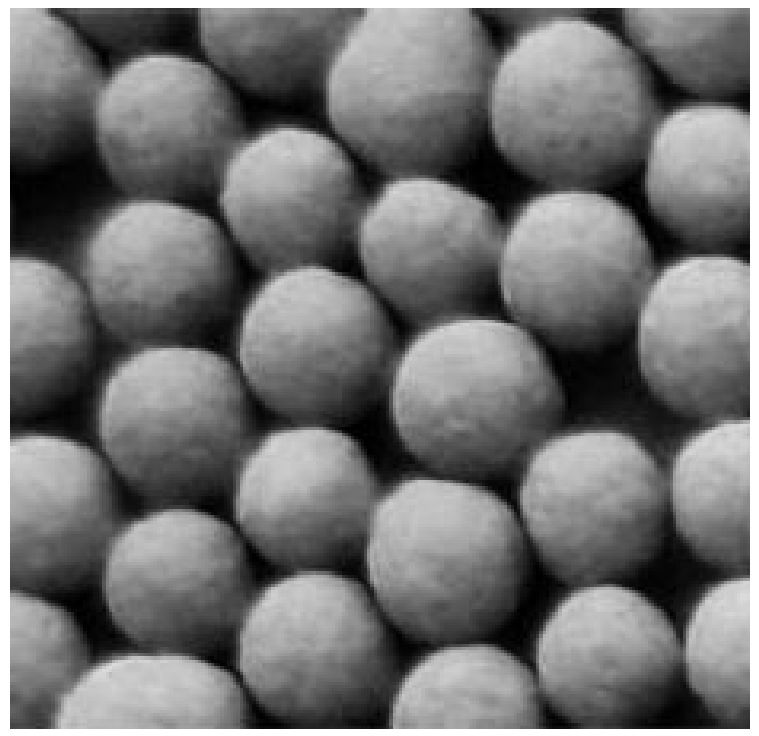

Figure 4. Bauxite, sphericity 0.90 - Photo courtesy: Oxane. 


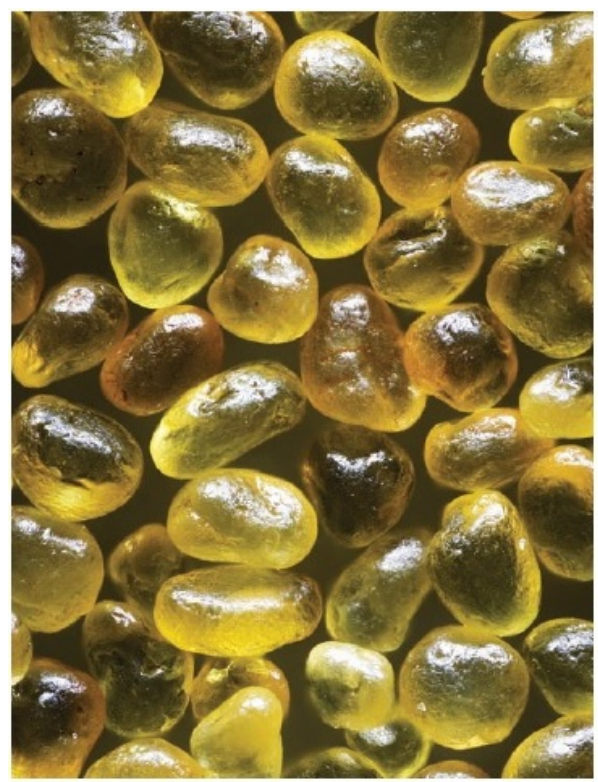

Figure 5. Resin Coated Sand, sphericity 0.80 - Photo courtesy: Santrol.

Figures 6 and 7 show a comparison of dimensionless permeability $\left(k / d_{p}^{2}\right)$ versus the characteristic proppant pack parameter, $\Omega$, for selected 20/40 Brown Sand (BS), 20/40 White Sand (WS), 20/40 resin coated sand, and 20/40 bauxite proppants at a concentration of $C_{a}=2 \mathrm{lb}_{m} / f t^{2}$. As illustrated, these proppants generally follow the correlation of Eq. 5 . However, the substantial permeability reduction as a result of the low sphericity is evident for the BS and to a lesser extent in the resin coated. The WS high permeability at about $\Omega=2 e-05$ is suspect (see Figure 7). 


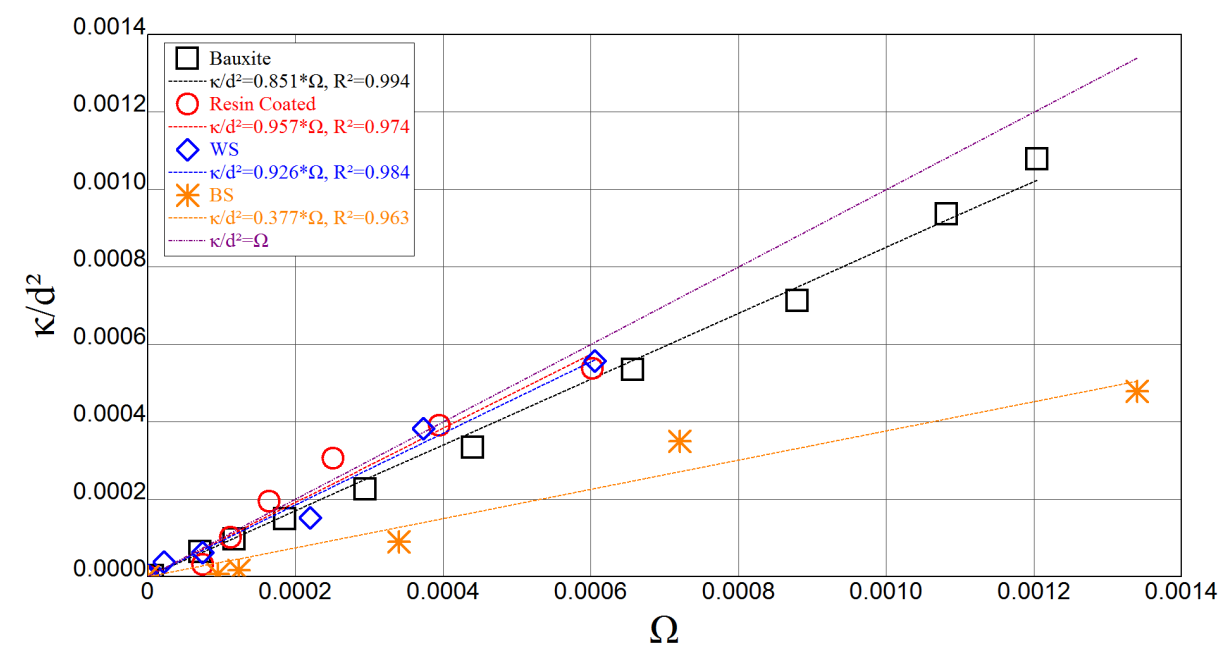

Figure 6. Correlation of Dimensionless Permeability for various types of 20/40 Proppants - Linear Plot.

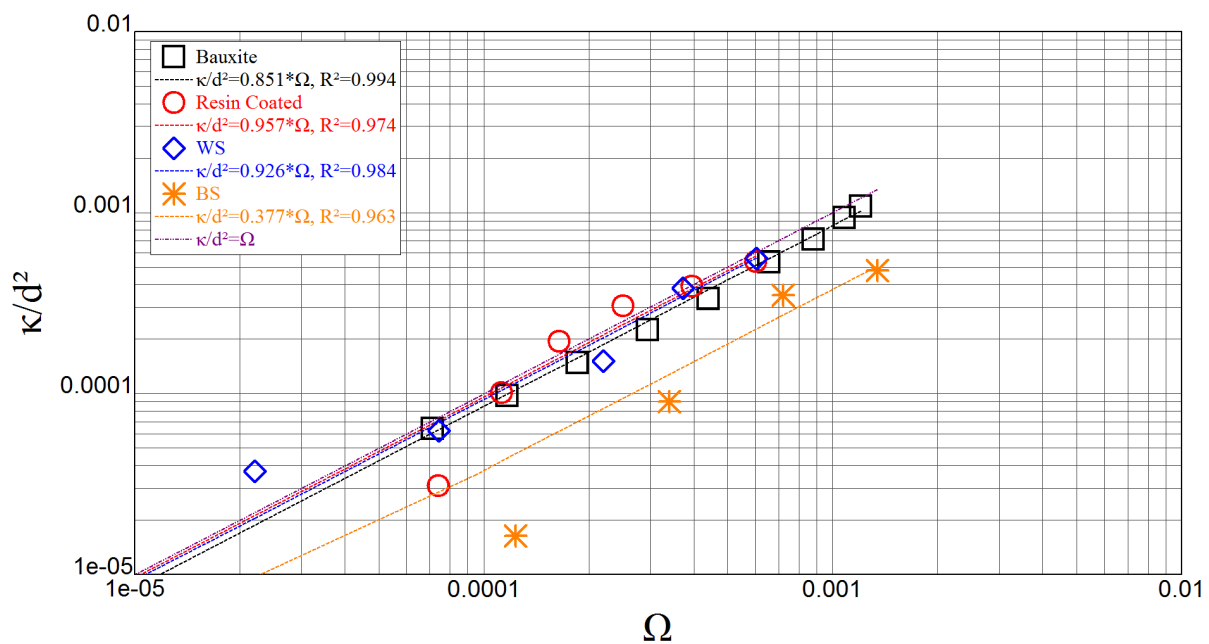

Figure 7. Correlation of Dimensionless Permeability for various types of 20/40 Proppants - Log-Log Plot. 


\section{Conclusion}

The fundamental solution for flow through packed columns (proppant packs) can be characterized by the sum of viscous and inertial forces (e.g., Ergun's equation). Coupling Ergun's equation with the Forchheimer equation results in a deterministic equation for the fracture permeability, $k_{f}$, and inertial factor, $\beta$, as functions of the proppant diameter, sphericity, pack porosity and width. Plotting the dimensionless permeability $k / d_{p}^{2}$ versus $\Omega$ can be a very useful diagnostic tool that can indicate: 1) sphericity, 2) channeling, 3) crushing, 4) non-uniform sphere size distribution, 5) embedment and 6) deviation of the friction multiplier $\lambda_{m}$ from Ergun's equation. Large deviations can also signify poor or inconsistent experimental results. This diagnostic plot can also quantify the behavior of proppant mono-layers.

\section{Nomenclature}

$a_{D}=$ Dimensionless specific surface area, $a_{D}=a_{w} / a_{s}$

$a_{s}=$ Specific surface area - sphere

$a_{w}=$ Specific surface area - wall

$A=$ Cross-sectional area

$A_{p}=$ Particle surface area

$C_{a}=$ Concentration/area

$d_{h}=$ Hydraulic diameter

$d_{p}=$ Proppant diameter

$d_{p}^{\prime}=$ Equivalent proppant diameter, $d_{p}^{\prime}=\Phi d_{p}$

$f=$ Darcy friction factor

$f_{0}=$ Burke-Plummer friction factor

$g=$ Gravitational constant

$k=$ Permeability

$K=$ Loss coefficient - inlet and exit

$L=$ Column length

$L_{\tau}=$ Tortuous path length

$N_{m l}=$ Number of minor losses 
$p=$ Pressure

$P_{f}=$ Perimeter

$q=$ Flow rate

$\operatorname{Re}=$ Reynolds number

$v=$ Superficial velocity

$\langle\bar{v}\rangle=$ Cross-sectional velocity

$V_{p}=$ Particle volume

$w=$ Width

\section{Greek}

$\beta=$ Inertial or beta factor

$\lambda_{m}=$ Friction factor multiplier

$\mu=$ Viscosity

$\tau_{w}=$ Shear stress - wall

$\phi=$ Porosity

$\Phi=$ Sphericity

$\Omega=$ Characteristic proppant porosity parameter

$\Psi=$ Sphericity-specific area parameter

$\rho=$ Density

\section{Subscripts}

$f=$ Fracture

$h=$ Hydraulic

$p=$ Proppant

$=$ Sphere

$w=$ Wall or width

\section{Appendix A: Flow through packed columns}

The solution methodology for flow through a proppant pack can be developed from flow through packed columns as presented by Bird, Stewart, and Lightfoot (1960). Although a 
detailed derivation of the equations for determining proppant permeability and inertial effects is not within the scope of this paper, the fundamentals are provided to give the reader an appreciation of the dominant parameters that affect the proppant pack permeability.

As discussed by Bird et al., "the packing material may be spheres, cylinders, or various other kinds of packing shapes. It is also assumed that the packing is everywhere uniform and that there is no channeling of fluid (in actual practice, channeling frequently occurs and the formulas provided are not valid). It is further assumed that the diameter of the packing is small in comparison with the diameter of the column in which the packing is contained and that the column diameter is constant." The impact of these last two assumptions will be addressed later in this section.

\section{Governing equations}

The governing equations for flow through packed columns are formulated in this section. Friction factors for packed columns, frictional pressure loss for laminar flow, and inertial flow (non-Darcy) are presented. Derivation for the fracture permeability and inertial coefficient $(\beta)$ are also presented.

\section{Friction factor}

The friction factor is normally defined as the ratio of friction forces to inertial forces. This factor is commonly used to determine the frictional dissipation in closed conduits and is defined as

$$
f=\frac{4 \tau_{w}}{1 / 2 \rho v^{2}}=\frac{2 d_{h}(-d p / d x)}{\rho v^{2}}
$$

where $f$ is the Darcy friction factor, $\tau_{w}$ is the wall shear stress, $v$ is the superficial velocity $(v=q / A)$, and $d_{h}$ is the hydraulic diameter. The pressure gradient in the conduit is $-d p / d x$. The hydraulic diameter in packed columns is sometimes replaced with the equivalent particle diameter or other characteristic dimension.

\section{Hydraulic diameter}

The hydraulic diameter is defined as

$$
d_{h}=\frac{4 A}{P_{f}}
$$

where $P_{f}$ is the conduit wetted perimeter and $A$ is the flow cross-sectional area.

\section{Laminar flow}

The equation of motion for laminar flow in closed conduits (e.g., pipes, slots, annuli and other non-circular conduits) can be represented by 


$$
-\frac{d p}{d x}=\frac{32 \lambda_{m} \mu\langle\bar{v}\rangle}{d_{h}^{2}}
$$

where the average flow rate in the cross section available for flow is given by the intrinsic velocity $\langle\bar{v}\rangle, \lambda_{m}$ is a friction factor multiplier that is a function of the closed conduit geometry, and $d_{h}$ is the hydraulic diameter. Theoretically, the friction multiplier for flow of a Newtonian fluid in a pipe, narrow elliptical slot, and rectangular slot are $\lambda_{m}=1, \lambda_{m}=\pi^{2} / 8$, and $\lambda_{m}=3 / 2$, respectively.

The pressure loss in terms of the Darcy friction factor based on the cross-sectional average flow velocity from Eq. A.3 is

$$
-\frac{d p}{d x}=\frac{f}{2} \frac{\rho\langle\bar{v}\rangle^{2}}{d_{h}}
$$

where the Darcy friction factor is given by

$$
f=\frac{64 \lambda_{m} \mu}{\rho\langle\bar{v}\rangle d_{h}}=\frac{64 \lambda_{m}}{\operatorname{Re}}
$$

The Reynolds number for flow of a Newtonian fluid in a conduit is defined as

$$
\operatorname{Re}=\frac{\rho\langle\bar{v}\rangle d_{h}}{\mu}
$$

where the cross-sectional average velocity $\langle\bar{v}\rangle$ is related to the superficial velocity $v$ by the conduit porosity (i.e., $\langle\bar{v}\rangle=v / \phi)$.

\section{Laminar flow in packed columns}

The frictional pressure loss through a proppant pack (or packed bed) can be derived from Eq. A.3

$$
-\frac{d p}{d x}=\frac{32 \lambda_{m} \mu\langle\bar{v}\rangle}{d_{h}^{2}}
$$

by replacing the cross-sectional average velocity $\langle\bar{v}\rangle$ by the superficial velocity $v$ (i.e., $\langle\bar{v}\rangle=v / \phi)$ and the equivalent hydraulic diameter of the proppant pack in terms of the particle diameter and porosity. 
The equivalent hydraulic diameter from Eq. A.2 for spherical particles with a diameter of $d_{p}$ and a packed porosity of $\phi$ is

$$
d_{h}=\frac{2}{3} \frac{\phi}{1-\phi} d_{p}
$$

Substituting the hydraulic diameter and the relationship $\langle\bar{v}\rangle=v / \phi$ into Eq. A.7, we find

$$
\begin{aligned}
-\frac{d p}{d x} & =\frac{32 \lambda_{m} \mu\langle\bar{v}\rangle}{d_{h}^{2}}=\frac{32 \lambda_{m} \mu(v / \phi)}{\left(\frac{2}{3} \frac{\phi}{1-\phi} d_{p}\right)^{2}} \\
& =\frac{72 \lambda_{m}(1-\phi)^{2} \mu v}{\phi^{3} d_{p}^{2}}
\end{aligned}
$$

Experimental measurements (Bird et al. 1960) indicate that if a frictional multiplier of $\lambda_{m}=25 / 12$ is used, the above theoretical equation matches extremely well with the experimental data. Insertion of this friction multiplier value into Eq. A.9 then gives

$$
-\frac{d p}{d x}=\frac{150(1-\phi)^{2} \mu v}{\phi^{3} d_{p}^{2}}
$$

which is the Blake-Kozeny equation. This equation is generally good for void fractions less than 0.5 and is valid in the laminar flow regime given by $\frac{(\rho v) d_{p}}{\mu(1-\phi)}<10$ (Bird 1960). The bed friction multiplier based on the proppant diameter is

$$
\begin{aligned}
f_{d_{p}} & =\frac{2 d_{p}(-d p / d x)}{\rho v^{2}} \\
& =\frac{144 \lambda_{m}}{\operatorname{Re}_{d_{p}}} \frac{(1-\phi)^{2}}{\phi^{3}}=\frac{300}{\operatorname{Re}_{d_{p}}} \frac{(1-\phi)^{2}}{\phi^{3}}
\end{aligned}
$$

where $\operatorname{Re}_{d_{p}}=\rho v d_{p} / \mu$. The bed friction factor based on the hydraulic diameter is 


$$
\begin{aligned}
f_{d_{h}} & =\frac{2 d_{h}(-d p / d x)}{\rho v^{2}} \\
& =\frac{64 \lambda_{m}}{\phi \operatorname{Re}_{d_{h}}}=\frac{400 / 3}{\phi \operatorname{Re}_{d_{h}}}
\end{aligned}
$$

where

$$
\operatorname{Re}_{d_{h}}=\rho v d_{h} / \mu
$$

\section{Inertial flow in packed columns}

The pressure loss in packed columns as a result of inertial forces (minor losses) was originally derived by Burke and Plummer assuming turbulent flow in packed columns (see Bird 1960). Burke and Plummer assumed that for highly turbulent flow that the friction factor was only a function of roughness and that the roughness characteristics were similar for all packed columns. Based on these assumptions Burke and Plummer could then justify a constant friction factor $f_{0}$ that would be used to characterize turbulent flow. Then from Eq. A.4 the resulting pressure loss equation for inertial losses is

$$
\begin{aligned}
-\frac{d p}{d x} & =\frac{f_{0}}{2} \frac{\rho\langle\bar{v}\rangle^{2}}{d_{h}} \\
& =\frac{3}{4} f_{0} \frac{\rho v^{2}}{d_{p}} \frac{1-\phi}{\phi^{3}}
\end{aligned}
$$

where the experimental data indicated that $f_{0} \cong 7 / 3$. This is the Burke-Plummer equation which is valid for $\frac{(\rho v) d_{p}}{\mu(1-\phi)}>1000$.

The form of the Burke-Plummer equation can also be derived assuming inertial forces (minor flow loss) through the proppant pack using the extended Bernoulli equation as presented below.

\section{Viscous and inertial flow in packed columns}

The flow through packed columns may also be characterized as a sum of frictional (viscous losses) and inertial (minor losses) forces. The general pressure loss equation formulation based on the extended Bernoulli equation with minor losses is

$$
\frac{p_{1}-p_{2}}{\rho g}=\sum f \frac{L_{\tau}}{d_{h}} \frac{\langle\bar{v}\rangle^{2}}{2 g}+\sum k \frac{\langle\bar{v}\rangle^{2}}{2 g}
$$


or

$$
-\frac{d p}{d x}=\sum f \frac{L_{\tau}}{L} \frac{\rho\langle\bar{v}\rangle^{2}}{2 d_{h}}+\sum K \frac{L_{\tau}}{L} \frac{\rho\langle\bar{v}\rangle^{2}}{2 d_{h}}
$$

where $L$ is the length of the column and $L_{\tau}$ is the tortuous path length the fluid takes. Further assume that the number of minor losses $N_{m l}$ in a column of length $L$ can be approximated by $N_{m l}=L_{\tau} / d_{h}$. Then the exit and entrance losses as the fluid expands and contracts through the packed column from Eq. A.15 can be written as

$$
\begin{aligned}
-\frac{d p}{d x} & =f \frac{L_{\tau}}{L} \frac{\rho\langle\bar{v}\rangle^{2}}{2 d_{h}}+\sum_{i=1}^{N_{m l}}\left(K_{\text {inlet }}+K_{\text {exit }}\right) \frac{\rho\langle\bar{v}\rangle^{2}}{2 d_{h}} \\
& =f_{d_{h}} \frac{\rho\langle\bar{v}\rangle^{2}}{2 d_{h}}+f_{0} \frac{\rho\langle\bar{v}\rangle^{2}}{2 d_{h}}
\end{aligned}
$$

The inertial pressure loss is identical to the form of the Burke-Plummer equation even though one was based on inertial effects and the other on turbulence. This, however, should not be surprising since both inertial and turbulent losses are proportional to $\rho v^{2}$. Substituting the bed friction factor and superficial velocity into Eq. A.16, we find

$$
-\frac{d p}{d x}=\frac{32 \lambda_{m} \mu v}{\phi d_{h}^{2}}+f_{0} \frac{\rho v^{2}}{2 \phi^{2} d_{h}}
$$

or

$$
-\frac{d p}{d x}=\frac{72 \lambda_{m}(1-\phi)^{2} \mu v}{\phi^{3} d_{p}^{2}}+\frac{3}{2} f_{0} \frac{1-\phi}{\phi^{3}} \frac{\rho v^{2}}{2 d_{p}}
$$

where from experimental data $\lambda_{m}=25 / 12$ and $f_{0}=7 / 3$.

\section{Ergun's equation}

The total pressure loss formulation for all flow regimes may thus be obtained by simply adding the Blake-Kozeny equation for viscous dissipation and the Burke-Plummer equation for inertial losses. The above equation can be written in terms of the dimensionless groups as follows 


$$
-\left(\frac{d p}{d x} \frac{d_{p}}{\rho v^{2}}\right)\left(\frac{\phi^{3}}{1-\phi}\right)=\frac{150(1-\phi)}{\operatorname{Re}}+\frac{7}{4}
$$

This is the Ergun equation (Bird 1960) where $\operatorname{Re}=\rho v d_{p} / \mu, \lambda_{m}=25 / 12$ and $f_{0}=7 / 3$ have been substituted.

\section{Darcy and non-darcy flow}

The equation to describe non-Darcy flow is a form of the Forchheimer (1901) equation

$$
-\frac{d p}{d x}=\frac{\mu}{k} v+\beta \rho v^{2}
$$

where $k$ is the permeability of the porous media and $\beta$ is the non-Darcy or inertial factor. Clearly, the first term in this equation accounts for viscous effects and the second term for inertial or minor loss effects.

Rearranging Eq. A.20 in terms of the dimensionless groups we find

$$
(-d p / d x) / \beta \rho v^{2}=1 / \operatorname{Re}_{\beta k}+1
$$

where the dimensionless Reynolds number for non-Darcy flow is given by

$$
\operatorname{Re}_{\beta k}=\frac{\rho v \beta k}{\mu}
$$

Rewriting Eq. A.17 in terms of the fracture permeability $k$ and inertial factor $\beta$ from Eq. A.20, we have

$$
\begin{aligned}
-\frac{d p}{d x} & =\frac{32 \lambda_{m} \mu v}{\phi d_{h}^{2}}+f_{0} \frac{\rho v^{2}}{2 \phi^{2} d_{h}} \\
& =\frac{\mu}{k} v+\beta \rho v^{2}
\end{aligned}
$$

where

$$
k=\frac{\phi d_{h}^{2}}{32 \lambda_{m}} \text { and } \beta=\frac{f_{0}}{2 d_{h} \phi^{2}}
$$


Placing $\beta$ in terms of the fracture permeability we find

$$
\beta=\frac{3 / 4 f_{0}}{\sqrt{72 \lambda_{m}}} \frac{1}{\sqrt{k \phi^{3}}}
$$

\section{Sphericity}

Sphericity is a measure of how closely a grain approaches the shape of a perfect sphere compared to roundness which is a measure of the sharpness of grain corners. The sphericity of a particle is the ratio of the surface area of a sphere (with the same volume as the given particle) to the surface area of the particle

$$
\Phi=\frac{\pi^{1 / 3}\left(6 V_{p}\right)^{2 / 3}}{A_{p}}
$$

where $V_{p}$ is the volume and $A_{p}$ is the surface area of the particle. For non-spherical particles the characteristic particle diameter in the above equations must be replaced by

$$
d_{p}^{\prime}=\Phi d_{p}
$$

\section{Proppant permeability}

The proppant permeability can be theoretically calculated from Eq. A.24 provided that the hydraulic diameter and porosity are known. The hydraulic diameter for flow in a slot of width $(w)$, packed with a proppant of uniform porosity $(\phi)$, and diameter $\left(d_{p}\right)$ from Eq. A.2 is

$$
d_{h}=\frac{4 \phi}{\left(a_{s}+a_{w}\right)}
$$

where the specific surface areas for the proppant spheres and fracture wall are $a_{s}=6(1-\phi) / d_{p}$ and $a_{w}=2 / w$, respectively.

The proppant permeability in terms of the proppant diameter, porosity, slot width, and sphericity is found by substituting Eq. A.26 through A.28 into A.24

$$
k=\frac{\phi^{3} \Phi^{2} d_{p}^{2}}{72 \lambda_{m}(1-\phi)^{2}}\left(1+\frac{\Phi d_{p}}{3(1-\phi) w}\right)^{-2}
$$


The permeability for flow through an open slot or channel (i.e., no proppant as $\phi \rightarrow 1$ ) with a slot width of $w$ from Eq. A.29 is

$$
k=\frac{9 w^{2}}{72 \lambda_{m}}=\frac{w^{2}}{12}
$$

where $\lambda_{m}=3 / 2$ for slot flow.

The dimensionless form of Eq. A.29 is

$$
\frac{k}{d_{p}^{2}}=\frac{\phi^{3} \Phi^{2}}{72 \lambda_{m}(1-\phi)^{2}}\left(1+\frac{\Phi d_{p}}{3(1-\phi) w}\right)^{-2}
$$

or

$$
\frac{k}{d_{p}^{2}}=\Omega \Psi
$$

where

$$
\begin{gathered}
\Omega=\frac{\phi^{3}}{72 \lambda_{m}(1-\phi)^{2}}\left(1+a_{D}\right)^{-2} \\
\Psi=\Phi^{2}\left(\frac{1+a_{D}}{1+\Phi a_{D}}\right)^{2} \\
a_{D}=\frac{a_{w}}{a_{s}}=\frac{d_{p}}{3(1-\phi) w}
\end{gathered}
$$

Eq. A.32 illustrates that the dimensionless permeability ratio $\left(k / d_{p}^{2}\right)$ is a linear function of the characteristic proppant pack parameter $(\Omega)$, and the proppant sphericity-specific surface area parameter $(\Psi)$ for all proppants. The dimensionless specific area ratio of the fracture (slot wall) and proppant is represented by $a_{D}$.

Pan et al. (2001) provides a good review of permeability versus porosity correlation for random sphere packing. Pan also proposed a modification to Ergun's equation for low Reynolds number with a four parameter fit model to correlate $k / d_{p}^{2}$ as a function of porosity and sphere size distribution. 


\section{Acknowledgements}

The authors wish to thank the management of Baker Hughes Incorporated for the opportunity to perform this research and publish the findings. We would also like to thank Bill Brinzer and Jennifer Pusch for their grammatical review and proofing of the manuscript.

\section{Author details}

Bruce R. Meyer ${ }^{1}$, Lucas W. Bazan² and Doug Walls ${ }^{3}$

*Address all correspondence to: lucas.w.bazan@bazanconsulting.com

1 Meyer Technologies, LLC, Natrona Heights, PA, USA

2 Bazan Consulting, Inc., Houston, TX, USA

3 Baker Hughes Incorporated, Natrona Heights, PA, USA

\section{References}

[1] API, American Petroleum Institute,: "Recommended Practices for Testing Sand Used in Hydraulic Fracturing Operations", API RP 56, March (1983).

[2] API, American Petroleum Institute,: "Recommended Practices for Testing Sand Used in Gravel Packing Operations", API RP 58, December (1995).

[3] API, American Petroleum Institute,: "Recommended Practices for Testing HighStrength Proppants Used in Hydraulic Fracturing Operations", API RP 60, December (1995).

[4] Bird, R. B, Stewart, W. E, \& Lightfoot, E. N. Transport Phenomena," Wiley, New York, (1960).

[5] Forchheimer, P. Wasserbewegung durch Boden. Zeitschrift des Vereines Deutscher" Ingenieuer, 45 edition, (1901).

[6] ISO, (2006). Amendment 13503-2: "Measurement of properties of Proppants used in Hydraulic Fracturing and Gravel-Packing Operations". ISO.org

[7] ISO, (2006). Amendment 13503-5: "Procedures for Measuring the Long-Term Conductivity of Proppants". ISO.org

[8] Much, M. G, \& Penny, G. S. Long-Term Performance of Proppants Under Simulated Reservoir Conditions," SPE/DOE 16415, May (1987). 
[9] Palisch, T, Duenckel, R, Bazan, L, Heidt, H, \& Turk, G. Determining Realistic Fracture Conductivity and Understanding its Impact on Well Performance". SPE 106301, January (2007).

[10] Palisch, T, Duenckel, R, Chapman, M, Woolfolk, S, \& Vincent, M. C. How to Use and Misuse Proppant Crush Tests- Exposing the Top 10 Myths". SPE 119242, January (2009).

[11] Pan, C, Hilpert, M, \& Miller, C. T. Pore-scale modeling of saturated permeabilities in random sphere packings," Physical Review E, November (2001). , 066702 
